\title{
Control of prosthetic hand by using mechanomyography signals based on support-vector machine classifier
}

\author{
Firas Saaduldeen Ahmed ${ }^{1}$, Noha Abed-Al-Bary Al-jawady ${ }^{2}$ \\ ${ }^{1}$ Department of Electrical Techniques, Technical Institute of Mosul, Northern Technical University, Iraq \\ ${ }^{2}$ Department of Electrical power Techniques Engineering, Technical Engineering College / Mosul, \\ Northern Technical University, Iraq
}

\begin{tabular}{l} 
Article Info \\
\hline Article histor \\
Received M \\
Revised Jul \\
Accepted Ju \\
\hline Keywords: \\
EMG \\
MMG \\
PR \\
RMS \\
SVM \\
WPT
\end{tabular}

Corresponding Author:

Firas Saaduldeen Ahmed

Department of Electrical Techniques

Technical Institute of Mosul

Northern Technical University, Iraq

Email: firas_saad@ntu.edu.iq

\begin{abstract}
Prosthetic devices are necessary to help amputees achieve their daily activity in the natural way possible. The prosthetic hand has controlled by type of signals such as electromyography (EMG) and mechanomyography (MMG). The MMG signals have represented mechanical signals that generate during muscle contraction. These signals can be detected by accelerometers or microphones and any kind of sensors that can detect muscle vibrations. The contribution of the current paper is classifying hand gestures and control prosthetic hands depends on pattern recognition through accelerometer and microphone are to detect MMG signals. In addition to the cost of prosthetic hand less than other designs. Six subjects are involved. In this present work is the devices. In this study, two of them are amputee subjects. Each subject performs seven classes of movements. Pattern recognition (PR) is used to classify hand gestures. The wavelet packet transform (WPT) and root mean square (RMS) as features extracted from the signals and Support vector machine (SVM) as a classifier. The average accuracy is $88.94 \%$ for offline tests and $84.45 \%$ for online tests. $3 \mathrm{D}$ printing technology is used in this study to build prosthetic hands.
\end{abstract}

This is an open access article under the CC BY-SA license.

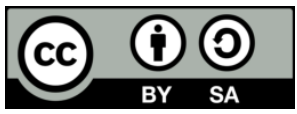

\section{INTRODUCTION}

Many people across the world have lost an upper limb due to a variety of factors such as wars, accidents, and illnesses. For these reasons, it has been necessary to develop a prosthetic device to help amputees for realization their activity naturally as possible. Recently the prosthetic hand has witnessed an improvement by using artificial intelligence such as pattern recognition [1], [2]. There are multi-modal sensing techniques that can be used to control prosthetic hand such as electromyography (EMG) which depend on the electrical activity of the muscle. This technique considers the most important and the most famous for classifying the hand gesture and control the prosthetic hand [3]. The other techniques such as, electroencephalography (EEG) which depend on the electrical activity of the brain but these signals are complicated and very hard to analyze [4], [5]. The other important signal is the mechanomyography (MMG) which represents the mechanical vibration of the muscles [6]. The EMG signals have many drawbacks, like the effect of electromagnetic disturbance from the surrounding device and affect by the sweat of the skin and electrode displacement [7], [8]. On the other MMG signals give a promising method for controlling 
prosthetic hand [8]. MMG signals can be detected by many kind of sensors such as accelerometers, microphone and piezoelectric sensors [9]-[12].

Many authors have studied the classification of hand movement depending on MMG signals Ding. 2017, investigated the detection of signals of the forearm from ten healthy subjects by using MMG sensors [13]. They used an IMU sensor (MPU 6050) with a pass-band filter. The features were extracted by using WPT (wavelet packet transform), SWT (stationary wavelet transform), and the time and frequency hybrid (TFDH) then classified by support vector machine (SVM) [14]. The research had found that WPT has the best performance [13]. The researchers investigated the hand gesture by using three dry gold-plated electrodes for EMG and MEMS accelerometer (AXDL203) for MMG signals [12]. A. Wołczowski and R. Zdunek, 2017, proposed an experimental method for measuring the EMG and MMG signals. The researcher used short time fourier transform (STFT) and discrete wavelet transform (DWT) to extract features of the received signals. It was acquired from eight electrodes placed on the forearm which is arranged in two rows upper and lower side on the forearm. The K-NN classifier was used to reduce and classify the features [15]. M. Fei et al. 2017, investigated the preference of using a hybrid system of MMG [13].

Ismail et al. 2019, studied the recognition of MMG signals which is collected by VMG sensor (TDS250A). The sensors were placed on palmaris longus-flexor carpi ulnaris (PL-FCU) and extensor digitorum-extensor carpi ulnaris (ED-ECU) muscles The experiments were conducted on eight healthy subjects. The signals were analyzed mean absolute value (MAV), standard deviation (STD), variance (VAR) and root mean square (RMS) and classified by LDA classifier [2]. Other research combine a system of EMG and MMG signals.

Nabeel. Siddiqui and Rosa. H. M, 2020, proposed a system of MMG by using ten microphones and one IMU sensors. The researchers have positioned the microphone and IMU sensors on the wrist. They have studied 13 gestures for ten trails for each set of gestures from 10 healthy subjects. They have used an SVM classifier to classify the gestures with overall accuracy equal to $80 \%$ and they found that the microphones compositions are not suitable [16].

This work aims to use the sensors (accelerometer and microphone) are to detect MMG signals for the prosthetic hand, which helps the personals amputees' hand at a low cost and good stability. The contribution of the current paper is classifying hand gestures and control prosthetic hand depends on pattern recognition. In addition to the cost of prosthetic hand less than other designs, were not more than the 40\$. Also, the usefulness of the present work is helping people with disabilities who have lost their limbs and have limited income because the proposed design is of relatively low cost.

In this paper, an accelerometer and microphone are to detect MMG signals. Six subjects are involved in this study two of them are amputee subjects. Each subject performs seven classes of movements. PR has been used to classify hand gestures. WPT and RMS as features extracted from the signals and SVM as classifiers. Also, 3D printing technology is used in this study to build prosthetic hands.

\section{PROPOSED APPROACH}

Proposed approach of the present work the classifying hand gestures and control prosthetic hands depends on pattern recognition through accelerometer and microphone are to detect MMG signals. The Six subjects are involved in this study four are healthy subjects, and the other two are trans-radial amputees. The sensors used in this study are accelerometers (MPU6050) and ADMP401 microphones for sensing MMG signals [7]. The location of the sensors is shown in Figure 1. The accelerometers are located in the opposite position under the elbow, The microphone, on the other hand, is positioned on the front side of the wrist.

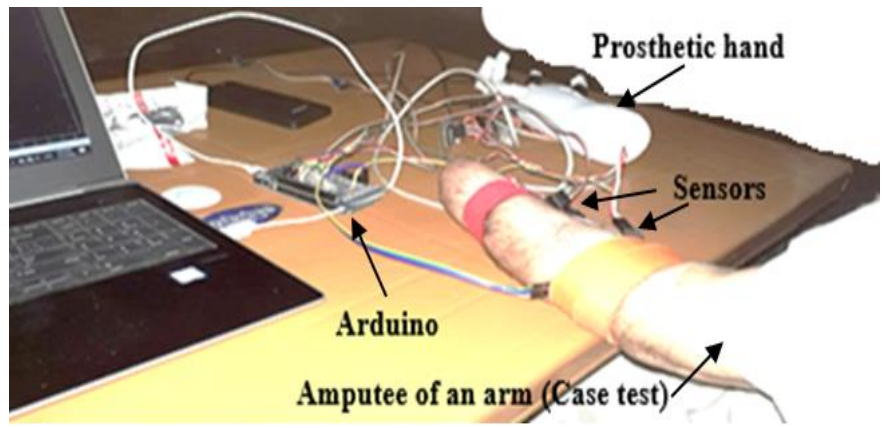

Figure 1. The sensors' location on the arm of the subject 


\subsection{Data acquisition and control system}

The signals are received from the sensors to be sent into Arduino MEGA then the Arduino sent the signals to the computer to process the signals by MATLAB 2018a, the MATLAB sent the order to another Arduino MEGA [17]. Figure 2 shows the method for connecting the sensors with Arduino due that connected to MATLAB connection of servos that control the prosthetic with Arduino Mega that connected to MATLAB [18].

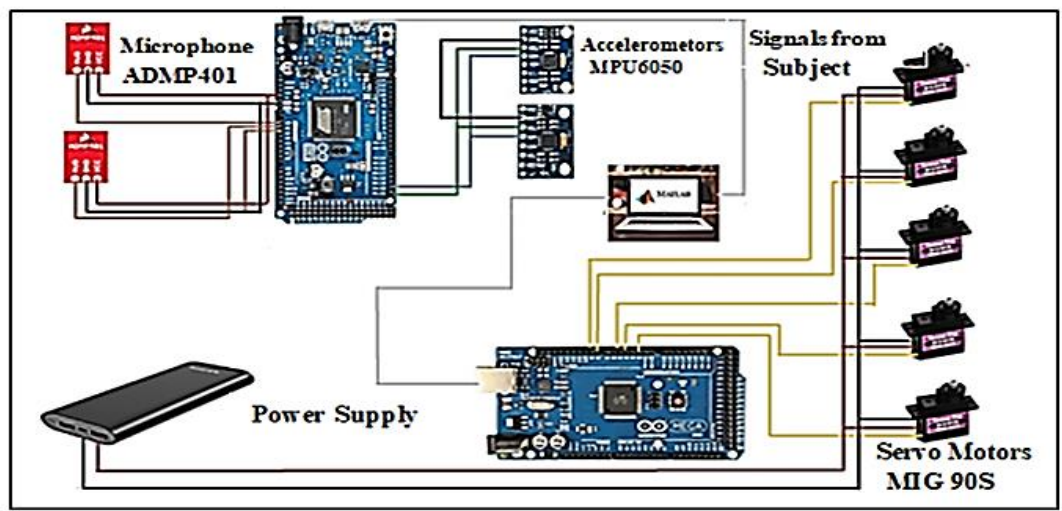

Figure 2. The connection method of MMG sensor and prosthetic hand servos with Arduino and MATLAB

The signals are represented 7 classes of movement which is 1 . Reset 2. Close 3. Open 4. Pinch 5. Tripod Pinch 6. Thump flexion 7. Index Flexion as shown in Figure 2 and the signals that comes from the sensor is shown in Figure 3. The signals that come from the sensor MMG signals for accelerometers and microphone sensors is shown in Figures 4 and 5 respectively [19].

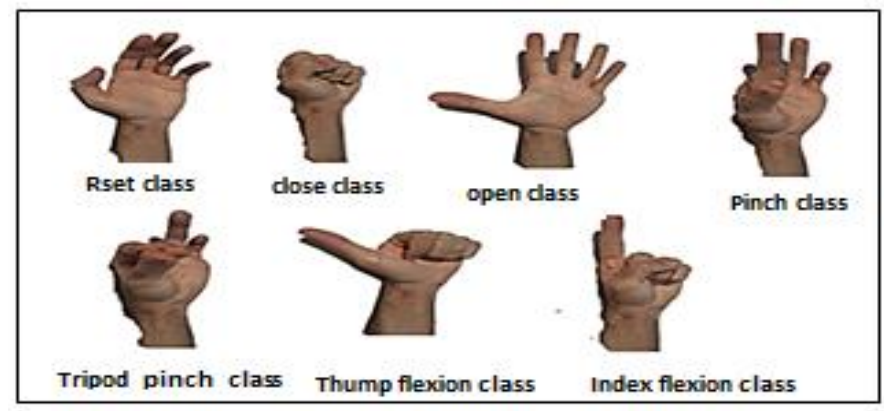

Figure 3. The hand gestures that depended in this study

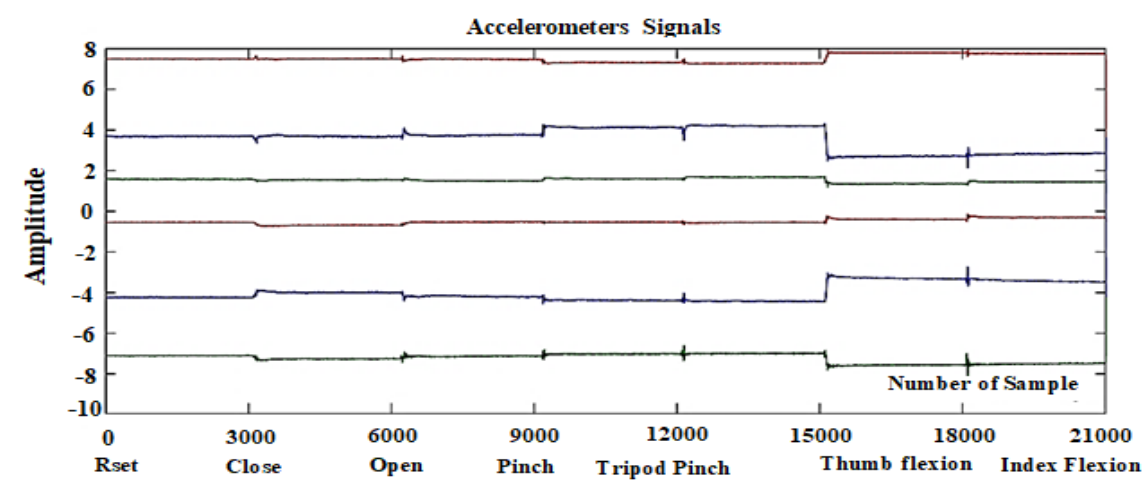

Figure 4. The sensor MMG signals for accelerometers 


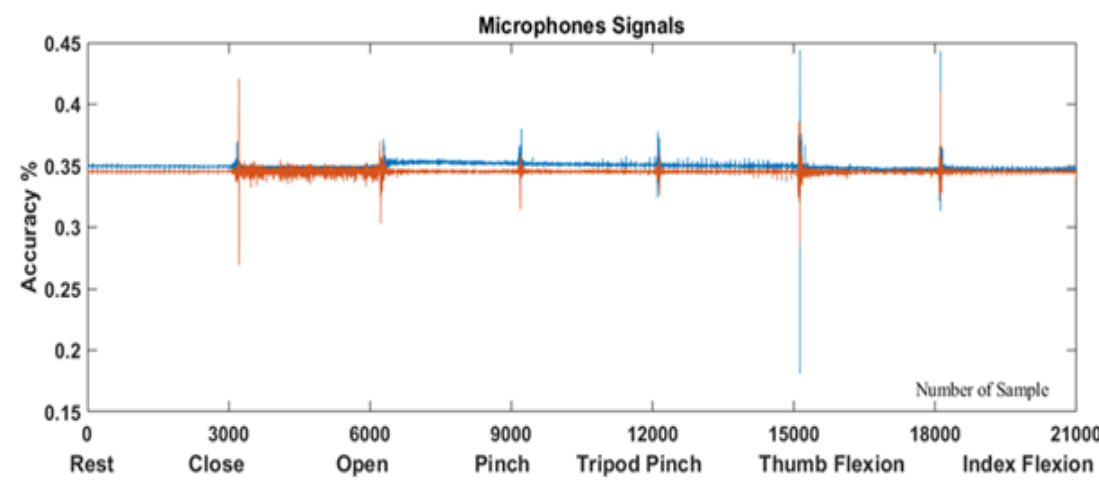

Figure 5. The sensors microphone signal

\subsection{Study algorithm}

This study is depending on pattern recognition as the main algorithm for analysis the signals and extract the hand gestures patterns [19]. PR is composed from many stages as shown in the Figure 6. These stages are:

- Filtering: the first stage is the filtering used to remove the unwanted signals or noise which accompanies the signals from multiple sources such as noise coming from the surrounding device or the noise from cross-talk that affects the microphones or noise from motion artifact that comes from unintentional movement. The filter used in this experiment was a second-order bandpass filter between 10-90Hz. Windowing (segmentation): The signals are segmented into multiple segments of time and perform the processing of feature extraction and classification on each window. The windowing type which is used in this case overlapping window. The window value used in this work is $256 \mathrm{~ms}$, with a $128 \mathrm{~ms}$ window increment.

- Feature extraction: Feature selection is the most important thing in the processing of pattern recognition. There are two types of features used in this study is root mean square (RMS), as shown in (1) [12], which consider one of the time domains features [20], and wavelet packet transform (WPT) that combine timedomain features and frequency domain features as shown in (2) [21], [22]. These features are timedomain features except for wavelet is time-frequency features.

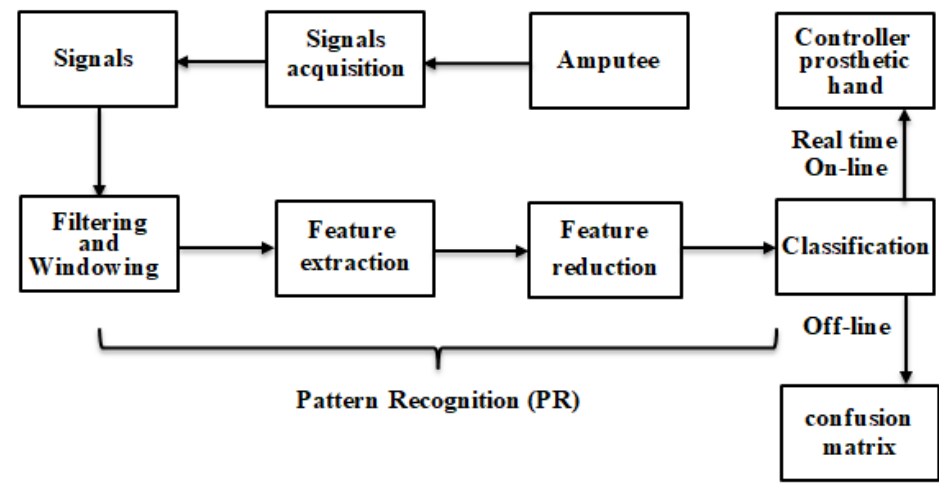

Figure 6. Pattern recognition stages was used in this study

$$
\mathrm{RMS}=\sqrt{\frac{1}{N} \sum_{i=1}^{N} x_{i}^{2}}
$$

Where: $\left(\mathrm{x}_{\mathrm{i}}\right)$ is the physiological signal and $(\mathrm{N})$ is the sample number.

$$
S(l)=\log \left(\frac{\sum_{m=1}^{\infty} w_{x}(l, m)^{2}}{N_{l}}\right)
$$

Where: $S(l)$ and $\left(w_{x}\right)$ are filter bank energy and wvelet packet transform of signal respectively, $(l)$ is subband frequency index, $\left(N_{l}\right)$ is the number of wavelet coefficients in the HF PD subband. 
- Feature reduction. Feature reduction is used to reduce the number of features that produced extraction, especially when using wavelet features which produced a large number of features. There are several types of feature reduction techniques but this experiment depends on principle component analysis (PCA). PCA is the most famous technique used in this kind of study [23], [24].

\subsection{Classifier}

The classification is the final stage of pattern recognition. The classifier must be trained with a set of training features and test it with testing features then make a comparison between the actual and predicted classes to make a judgment on the system response. The classifier has used in this study is the SVM classifier. The support vector machine can be a linear or non-linear classification that has been used to classify non-linear categories. This classifier implements classification by predicting the best hyper-plane, which separates the class data from other classes. A better hyper-plan can be found by finding a greater margin between the two classes. The maximal width of the plane that parallels to hyper-plan can define as the largest margin [25], [26]. The linear SVM has dependent on this study.

\subsection{Offline test}

In offline test, each subject is told to perform six set of movements and each set of movements is consist of seven class of movements that describe in the Figure 3. Every movement takes 3 seconds so that the set takes 21 seconds. Four sets of movement have been used to train the SVM classifier, and the other two sets have been used to test the classifier. The offline test has used to evaluate the signals and show the best position for the sensors on the hand. The importance of the offline test has evaluated the study algorithm and how to make the best accuracy [27].

\subsubsection{Online test}

In the online test, each subject controls the prosthetic hand in a real-time state with specified classes described in Figure 2. As mentioned in the offline test, four sets have also used to train the classifier then the trained classifier is used to control the prosthetic hand. Then each subject performs the specified gesture, which will directly control the prosthetic hand to calculate the accuracy [28].

\section{PROSTHETIC HAND}

The challenge of the present work is how can build design work with good efficiency and at a low cost. The prosthetic hand is designed based on InMoov's design [29], [30]. The InMoov designs are open source which permits modification of the circuit. The circuits are suitable for many levels of amputations, such as above-elbow amputee (trans-humeral) and below-elbow amputee (trans-radial), as shown in Figure 7.

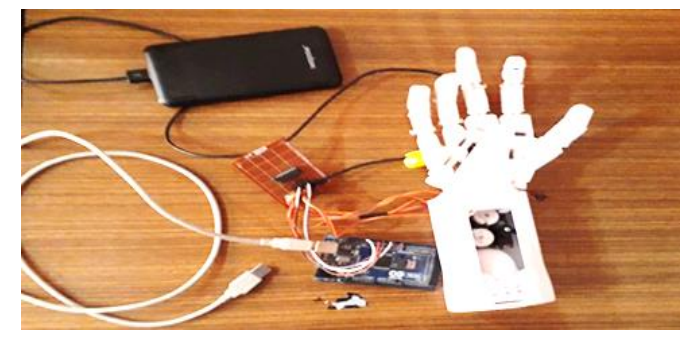

Figure 7. The control system for prosthetic hand

\section{RESULTS AND DISCUSSION}

The result of the six subjects is shown in Table 1 for offline tests and online tests, where the average accuracy for the offline test is $88.94 \%$, and the average accuracy for online tests is $84.45 \%$. The signal accuracy for amputee subjects is lower than the healthy subject because their muscles are weaker at the amputated limbs and this reason has affected the accuracy of the read healthy subject. The nerves of healthy people receive signals quickly because they are not exposed to an amputation accident. After all, the person sees the hand in front of him correctly. Therefore, it has self-confidence that is greater than that of amputees.

The other reason that affects the accuracy is that some subjects repeat the experiment more than one time. Therefore leads to making the person more Skill than the ordinary person and could perform the correct gesture in less time. And the error during the record of the signals is diminished. There is a potential reason that affects the result which is some subject are athletic than the other, and their muscles are stronger which make the response of the sensor better, therefore muscle fatigue may have low effect on athletic subjects. 
Table 1. The average accuracy for offline and online tests

\begin{tabular}{ccc}
\hline Subject & Accuracy for offline tests & Accuracy for online test \\
\hline Amputee & $86.54 \%$ & $80.75 \%$ \\
Amputee & $85.93 \%$ & $81.90 \%$ \\
Amputee & $92.97 \%$ & $83.49 \%$ \\
Amputee & $89.60 \%$ & $88.38 \%$ \\
Amputee & $90.21 \%$ & $83.49 \%$ \\
Amputee & $88.38 \%$ & $88.69 \%$ \\
\hline
\end{tabular}

Figures 8 and 9 shown the 1 and 2 samples of the subject for the confusion matrix for amputee at offline test, they have been taken six subject tests when offline and online, therefore it is worth noting the results were summarized as mentioned in the above table. The requirement of the present work has implemented when compared with an online test and an offline test. Also, the results show in the Table 1, the validated where the online test less than accuracy offline test because the response of microphone and accelerometer, the Hand nerve response. The average accuracy for offline tests is higher than the online because the hardware limitation as shown in Figure 10. The comparison between online and offline accuracy from the literature review with present work accuracy results is obtained in Table 2.

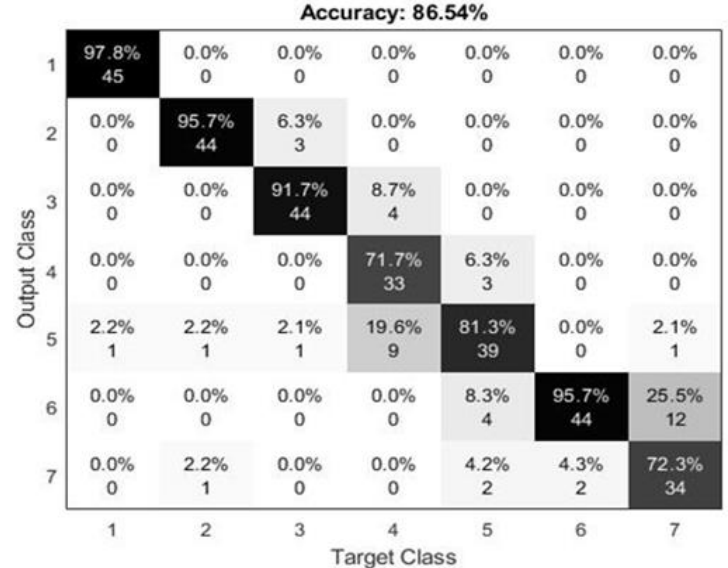

Figure 8. Confusion matrix for amputee subject in offline test

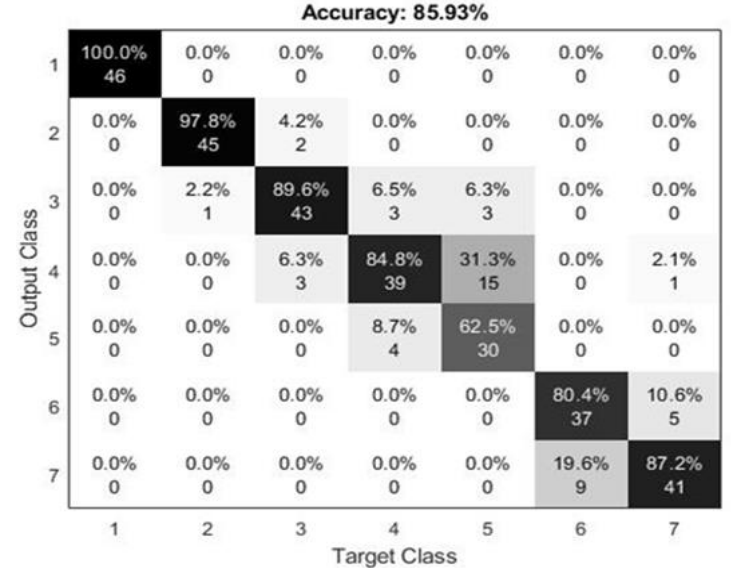

Figure 9. Confusion matrix for amputee subject in offline test

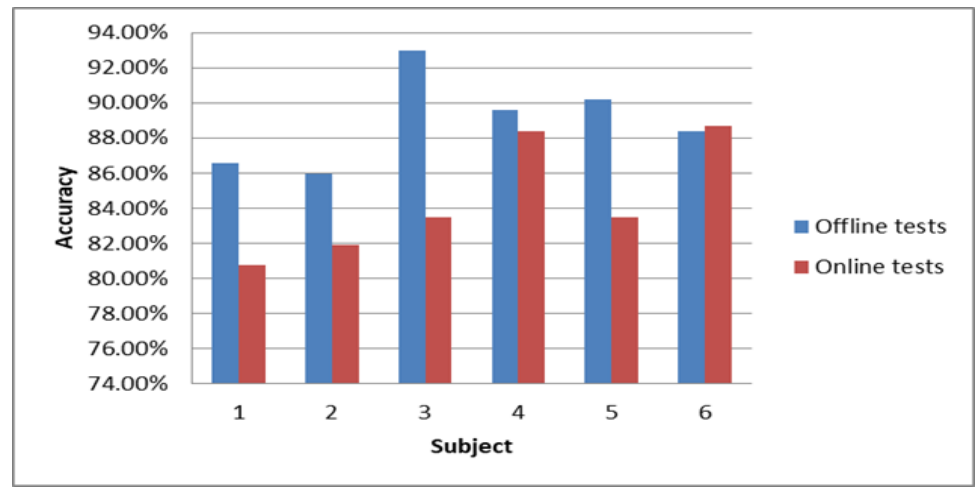

Figure 10. Comparison between offline and online accuracy

Table 2. The comparison between present work and literature review

\begin{tabular}{lccc}
\hline \multicolumn{1}{c}{ Reference with present work } & Accuracy offline & Accuracy online & cost \\
\hline Fei, Ma, and Li, 2017 & $86.41 \%$ & $82.53 \%$ & High cost \\
Ismail et al. 2019 & $87.5 \%$ & $83.34 \%$ & High cost \\
Nabeel. Siddiqui and Rosa. H. M, 2020 & $88 \%$ & $80 \%$ & High cost \\
Present work & $88.94 \%$, & $84.45 \%$ & Less cost approximatly $40 \$$ \\
\hline
\end{tabular}




\section{CONCLUSION}

The MMG signals have taken from the hand of six subjects where two of them is amputee subjects hand each subject performs seven gestures depend on pattern recognition. The wavelet packet transform and RMS are used as features in Pattern Recognition, and the Support vector machine is the classifier for hand gestures. The signals have been used to control a five-degree of $3 \mathrm{D}$ printing prosthetic hand. The results showed of the offline test has shown better accuracy than the online test. Also, the accuracy of results in the present work is better than the results accuracy of the literature reviews. In addition, this system has been considered low-cost compare to the other prosthetic hand because the devices used (Arduino, sensors, and servo motor) are low-cost and at the same time gave good results. It is important to note, the cost of a prosthetic hand less than others, approximately $40 \$$. Also, the same prosthetic hand can be improved performance by adding other kinds of sensors like electromyography (EMG) to more enhance the accuracy.

\section{ACKNOWLEDGEMENTS}

The authors want to express to thank the Northern Technical University, Iraq/ Mosul Technical Institute for their assistance, which helped advance this work.

\section{REFERENCES}

[1] A. Cloutier and J. Yang, "Control Of Hand Prostheses- A Literature Review," ASME 2013 International Design Engineering Technical Conferences and Computers and Information in Engineering Conference, 2013, pp. 1-11, doi: 10.1115/DETC2013-13349.

[2] M. R. Mohamad Ismail, C. K. Lam, K. Sundaraj, and M. H. F. Rahiman, "Hand motion pattern recognition analysis of forearm muscle using MMG signals," Bulletin of Electrical Engineering and Informatics, vol. 8, no. 2, pp. 533-540, 2019, doi: 10.11591/eei.v8i2.1415.

[3] J. Too, A. R. Abdullah, T. N. S. T. Zawawi, N. M. Saad, and H. Musa., "Classification of EMG Signal Based on Time Domain and Frequency Domain Features," International Journal of Human and Technology Interaction, vol. 1, no. 1, pp. 1-7, 2017.

[4] D. Tkach, H. Huang, and T. A. Kuiken, "Study of stability of time-domain features for electromyographic pattern recognition," Journal of Neuroengineering and Rehabilitation, pp. 1-13, 2010, doi: 10.1186/1743-0003-7-21.

[5] M. Mohammadi, F. Al-Azab, B. Raahemi, G. Richards, and N. Jaworska, "Data mining EEG signals in depression for their diagnostic value Clinical decision-making, knowledge support systems, and theory," BMC Med. Inform. Decis. Mak., vol. 15 no. 1, 1-14, 2015, doi: 10.1186/s12911-015-0227-6.

[6] Y. Y. Fang, N. Hettiarachchi, D. Zhou, and H. Liu, "Multi-modal sensing techniques for interfacing hand prostheses: A review," IEEE Sens. J., vol. 15 no. 11, pp. 6065-6076, 2015, doi: 10.1109/JSEN.2015.2450211.

[7] A. Testa, M. Cinque, A. Coronato, G. De Pietro, and J. C. Augusto, "Heuristic strategies for assessing wireless sensor network resiliency: an event-based formal approach," Journal of Heuristics, vol. 21, no. 2, pp. 145-175, 2014, doi: 10.1007/s10732-014-9258-x.

[8] D. Esposito, E. Andreozzi, A. Fratini, G. D. Gargiulo, and S. Savino, "A piezoresistive sensor to measure muscle contraction and mechanomyography," Sensors Switzerland, vol. 18 no. 8, pp. 1-12, 2018, doi: 10.3390/s18082553.

[9] R. de la Rosa, A. Alonso, A. Carrera, R. J. Durán, and P. Fernández, "Man-machine interface system for neuromuscular training and evaluation based on EMG and MMG signals,” Sensors, no. 12, pp. 11100-11125, 2010, doi: $10.3390 / \mathrm{s} 101211100$.

[10] M. O. Ibitoye, N. A. Hamzaid, J. M. Zuniga, and A. K. A. Wahab, "Mechanomyography and Muscle Function Assessment: A Review of Current State and Prospects," Clinical Biomechanics, vol. 29, no. 6, pp. 691-704, 2014, doi: 10.1016/j.clinbiomech.2014.04.003.

[11] C. Berceanu and D. Tarnita, "Mechanical Design and Control Issues of A Dexterous Robotic Hand," Advanced Materials Research, vol. 463-464, pp. 1268-1271, 2012, doi: 10.4028/www.scientific.net/AMR.463-464.1268.

[12] W. Guo, X. Sheng, H. Liu, and X. Zhu, "Mechanomyography assisted myoeletric sensing for upper-extremity prostheses: A hybrid approach," IEEE Sensors Journal, vol. 17 no. 10, pp. 3100-3108, 2017, doi: 10.1109/JSEN.2017.2679806.

[13] M. Fei, S. Ma, and X. Li, "Advanced Computational Methods in Life System Modeling and Simulation," Communications in Computer and Information Science, vol. 761, pp. 23-34, 2017, doi: 10.1007/978-981-10-6370-1.

[14] Li Zhang, Weida Zhou, and Licheng Jiao, "Wavelet support vector machine," IEEE Transactions on Systems, vol. 34, no. 1, pp. 34-39, 2004, doi: 10.1109/TSMCB.2003.811113.

[15] A. Wołczowski, and R. Zdunek, "Electromyography and mechanomyography signal recognition: Experimental analysis using multi-way array decomposition methods," Biocybern. Biomed. Eng, vol. 37, no. 1, pp. 103-113, 2017, doi: 10.1016/j.bbe.2016.09.004.

[16] N. Siddiqui and R. H. M. Chan, "Multimodal hand gesture recognition using single IMU and acoustic measurements at wrist," PLoS One, vol. 15, no. 1, pp. 1-12, 2020, doi: 10.1371/journal.pone.0227039.

[17] H. M. Marhoon, A. Murad, and O. Bayat, "Implementation of rover tank firefighting robot for closed areas based on arduino microcontroller," Indonesian Journal of Electrical Engineering and Computer Science, vol. 21, no. 1, pp. 56-63, 2021, doi: 10.11591/ijeecs.v21.i1.pp56-63. 
[18] A. N. Hussain, A. J. Ali, and F. S. Ahmed, "Comparison and Evaluation between Two Hybrid Systems Using Renewable Sources and DSTATCOM," 6 th International Engineering Conference Sustainable Technology and Development, (IEC2020), Erbil, Iraq, pp. 73-79, 2020, doi: 10.1109/IEC49899.2020.9122934.

[19] S. Nurmaini, A. Zarkasi, D. Stiawan, B. Y. Suprapto, and S. D. Siswanti, "Robot movement controller based on dynamic facial pattern recognition," Indonesian Journal of Electrical Engineering and Computer Science, vol. 22, no. 2, pp. 125-135, 2021, doi: 10.11591/ijeecs.v22.i2.pp733-743.

[20] Samsuryadi, R. Kurniawan, and F. S. Mohamad, "Automated handwriting analysis based on pattern recognition: A survey," Indonesian Journal of Electrical Engineering and Computer Science, vol. 22, no. 1, pp. 196-206, 2021, doi: 10.11591/ijeecs.v22.i1.pp196-206.

[21] H. Zhou and G. Alici, "A compact and cost-effective pattern recognition based myoelectric control system for robotic prosthetic hands," IEEE/ASME International Conference on Advanced Intelligent Mechatronics (AIM), Boston, USA (Virtual Conference), 2020, pp. 270-275, doi: 10.1109/AIM43001.2020.9158830.

[22] R. N. Khushaba, A. Al-Jumaily, and A. Al-Ani, "Novel Feature Extraction Method based on Fuzzy Entropy and Wavelet Packet Transform for Myoelectric Control," nternational Symp. Commun. Inf. Technol, vol. 1, no. 7, pp. 352-357, 2007, doi: 10.1109/ISCIT.2007.4392044.

[23] Z. Y. Hasan, R. H. Altaie, and H. A. Al. Hassan, "Fusion for medical image based on discrete wavelet transform coefficient," Indonesian Journal of Electrical Engineering and Computer Science, vol. 21, no. 3, pp. 1407-1416, 2021, doi: 10.11591/ijeecs.v21.i3.pp1407-1416.

[24] S. Negi, Y. Kumar, and V. M. Mishra, "Feature extraction and classification for EMG signals using linear discriminant analysis," 2016 2nd International Conference on Advances in Computing, Communication, \& Automation (ICACCA) (Fall), 2016.

[25] G. R. Naik, S. E. Suviseshamuthu, M. Gobbo, A. Acharyya, and H. T. Nguyen, "Principal Component Analysis Applied to Surface Electromyography: A Comprehensive Review," IEEE Access, vol. 4, no. 1, pp. 4025-4037, 2016, doi: 10.1109/ACCESS.2016.2593013.

[26] M. A. Oskoei, and H. Hu, "Support vector machine-based classification scheme for myoelectric control applied to upper limb," IEEE Trans. Biomed. Eng, vol. 55, no. 8, pp. 1956-1965, 2008, doi: 10.1109/TBME.2008.919734.

[27] A. Krasoulis, S. Vijayakumar, and K. Nazarpour, "Effect of User Practice on Prosthetic Finger Control with an Intuitive Myoelectric Decoder," Frontiers in Neuroscience, vol. 13, pp. 1-3, 2019, doi: 10.3389/fnins.2019.00891.

[28] Y. Wu, S. Liang, L. Zhang, Z. Chai and C. Cao., "Gesture recognition method based on a single-channels EMG envelope signal," EURASIP Journal on Wireless Communications and Networking, vol. 3 no. 12, pp. 1-8, 2018, doi: 10.1186/s13638-018-1046-0.

[29] F. Aggogeri, T. Mikolajczyk, and J. O'Kane, "Robotics for rehabilitation of hand movement in stroke survivors," Advances in Rehabilitation Engineering with Robotics and Mechatronic Devices, vol. 4, no. 11, pp. 1-14, 2019, doi: 10.1177/1687814019841921.

[30] F. Aggogeri, "Special Collection on advances in rehabilitation engineering with robotics and mechatronic devices," Advances in Mechanical Engineering, vol. 12, no. 8, pp. 1-3, 2020, doi: 10.1177/1687814020949086.

\section{BIOGRAPHIES OF AUTHORS}

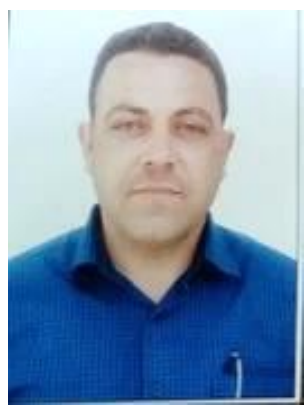

Firas Saaduldeen Ahmed was born in Mosul city, Nineveh, Iraq in 1984. he received a B.S degree in electrical power engineering from the Technical Engineering College Mosul in 2007 and a Master of the same field from the Electrical Engineering Technical College of Bagdad/Iraq in 2020. From 2021, are taught electrical machines, digital, electronics, and computer (IoT) at Mosul Technical Institute / Northern Technical University of Iraq. He has four research in a field electrical power and computer engineering and modern algorithms.

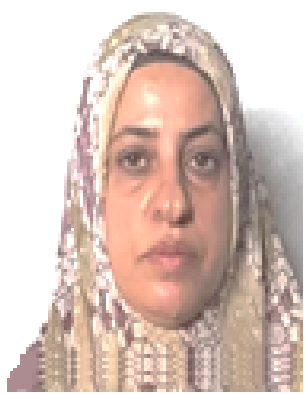

Noha Abed-Al-Bary Al-jawady was born in Mosul, Iraq in 1972, She received an M. Sc degree in electrical engineering from the college engineering electrical engineering department in the University of Mosul, Iraq, in 1999. After graduation, she joined the Northern Technical University, Mosul, Iraq, Technical engineering college of Mosul, as a Lecturer. She has much research in the field of protection power systems, Renewable energy, and A.C. Machine. 